\title{
Flaw Detection in Ceramics using Sigma Fuzzy Binarization and Gaussian Filtering Method
}

\author{
Kwang Baek Kim ${ }^{1}$, Doo Heon Song ${ }^{2}$ and Won Joo Lee ${ }^{3 *}$ \\ ${ }^{1}$ Department of Computer Engineering, Silla University, Korea \\ ${ }^{2}$ Department of Computer Games, Yong-in SongDam University, Korea \\ ${ }^{3}$ Department of Computer Science, Inha Technical College, Korea \\ gbkim@silla.ac.kr,dsong@ysc.ac.kr,wonjoo2@inhatc.ac.kr \\ Abstract
}

In this paper, we propose an intelligent method to extract flaws from nondestructive testing(NDT) images of ceramics. Our goal is to extend the preyious tudy[8]'s feasibility to handle images having skewed brightness distribution or darker)images. Thus, we use Gaussian filtering along with anisotropic filtenting and sigma fuzzy binarization instead of repetitive binarization used in [8]. These techriques enable us to enhance the brightness contrast successfully so that the usability of our method is extended and verified in experiment. Two phases of image processing - area segmentation and extraction of the defect by searching for labeled pixel to form a defected object by Grassfire algorithm - are explained in detail.

Keywords: Ends-in Search Stretching, Gamssian Filtering, Fuzzy Binarization, Ceramics

\section{Introduction}

Nondestructive Testing(NDT) refers to a group of analysis techniques to obtain necessary information from a material or a product without damaging its functional of shaping properties. Growing needs lor those techniques in recent years is mainly for the reliable quality assurance mechanisms and detect flaws of such materials/products.

There are so many avallable NDT methodologies such as radiographic testing, ultrasonic testing, magnetic resonance imaging(MRI), penetrant testing, eddy current testing, leak testing, acoustic emission testing, visual inspection, neutron testing, etc. according to the application areas and material types.

For the ceramic industry, the penetrant nondestructive testing is common in use [1]. Penetrant nondestructive testing(PT) is a widely-used inexpensive inspection method for detecting defects on the surface level of a material such as ceramics.

It applies liquid penetrant on the surface of the testing material and after sufficient dwelling time of the liquids to penetrate into discontinuing parts on its surface, applies white developer to soak penetrants in the discontinuing parts with removing excessive liquids on the surface so that one can visually inspect the size, location, and shape of discontinued parts that is mostly appeared to be the defects of the tested material. However, the process of visual inspection often causes subjective decision and relies on the experience of the inspector thus the reliability of such test might be affected and often time-consuming and expensive. Intelligent image analysis is a method for compensating such disadvantage of visual inspection.

${ }^{*}$ Corresponding Author 
Taking PT image as an input, a series of image processing techniques are applied to obtain necessary edge information and then usually a type of machine learning strategy is applied to determine the flaws on the testing material. A study successfully finds defects from ceramic tile surfaces by fuzzy thresholding technique [2] and another study applies Expectation Maximization (EM) algorithm to find appropriate threshold from ultrasonic image [3] or MRI image [4].

While intelligent, there is another approach to solve detecting defects of ceramic surface by image analysis. During the image processing process, it is helpful to enhance the image brightness to find a clear difference between the targeting edge and its neighbor areas. Thus a study uses anisotropic filtering mask and repetitive binarization to enhance the image brightness [5]. Unfortunately, this method is vulnerable when the input image has skewed brightness distribution. That method was usually successful but could not detect flaws from such unevenly distributed input image.

Thus, in this paper, we apply Gaussian Mask Filtering along with anisotropic filtering mask and choose Sigma fuzzy binarization instead of repetitive binarizationin order to handle such images having unevenly distributed brightness. In our.method, the whole process can be divided into two parts - area segmentation and flaw detection. Section 2 is devoted to explain the area segmentation, followed by flaw detection methodology in Section 3 .

\section{Area Segmentation by Slope Analysis}

There exists a boundary line in NDT image due to brightness differences. However, most parts of that line overlaps with defectedge so that detecting such an edge is not sufficient to find defect from the image. Thus we first extract the boundary area with $7 \times 7$ Sobel mask.

\subsection{Image Transform by Ends-in Search Stretching}

In order to enhance the brightness contrast of the input ceramic image from NDT, we apply Ends-in Search Stretching as shown in formula (1) below [6, 7]

$$
\text { outImg }[x][y]=\left\{\begin{array}{l}
0, \quad \text { for } X \leq \text { Low } \\
\frac{X-\text { Low }}{\text { High }- \text { Low }} \\
255, \text { for High } \leq X
\end{array} \times 255, \quad \text { for Low } \leq X \leq\right. \text { High }
$$

where $X$ denbtes the brightness value of current pixel and High and Low denote the highest and lowest brightness value of the area in respectively. Figure 1 shows the result of applying Ends-in Search Stretching compared with the original input image.

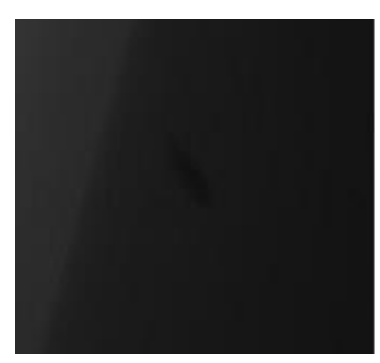

(a) Original image

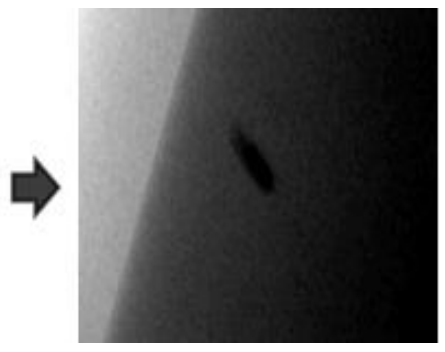

(b) After applying Ends-in stretching

Figure 1. Effect of Ends-in search stretching 


\subsection{Removing Minute Noise by Anisotropic Mask and Gaussian Filtering}

Anisotropic mask filtering applies different values to each upper/lower/left/right pixel of the current pixel under formula (2) below.

$$
\begin{gathered}
I_{i, j}^{t+1}=I_{i, j}^{t}+\lambda\left[c_{N} \cdot \nabla_{N} I+c_{S} \cdot \nabla_{S} I+c_{E} \cdot \nabla_{E} I+c_{W} \cdot \nabla_{W} I\right] \\
\nabla_{N}\left(I_{i, j}\right)=I_{i, j-1}-I_{i, j} \quad \nabla_{w}\left(I_{i, j}\right)=I_{i, j+1}-I_{i, j} \\
\nabla_{E}\left(I_{i, j}\right)=I_{i+1, j}-I_{i, j} \quad \nabla_{w}\left(I_{i, j}\right)=I_{i+1, j}-I_{i, j}
\end{gathered}
$$

where $0 \leq \lambda \leq 0.25$ for the mathematical convergence and denotes processing time and denotes current pixel. Symbols $N, S, E, W$ represents the north, south east, west direction.

Thus $\nabla_{N-w} I$ means first derivative of $I_{i, j}^{t}$ with directions and $C_{N-W}$ denotes the transferring coefficient function that controls each pixel's value in anisotropic mask filtering.

Then we apply Gaussian Mask Filtering with the image obtained from Ends-in Search Stretching according to the formula (3) explained below.

$$
G_{\sigma}(x, y)=\frac{1}{2 \pi \sigma^{2}} e^{\left(-\frac{x^{2}+y^{2}}{2 \sigma^{2}}\right)}=\frac{1}{\sqrt{2 \pi} \sigma} e^{\left(-\frac{x^{2}}{2 \sigma^{2}}\right)} \times \frac{1}{\sqrt{2 \pi} \sigma} e^{\left(-\frac{y^{2}}{2 \sigma^{2}}\right)}
$$

With above two mask filtering processes, we can remove minute noises and obtain finer image for further processing as shown in Figure 2.

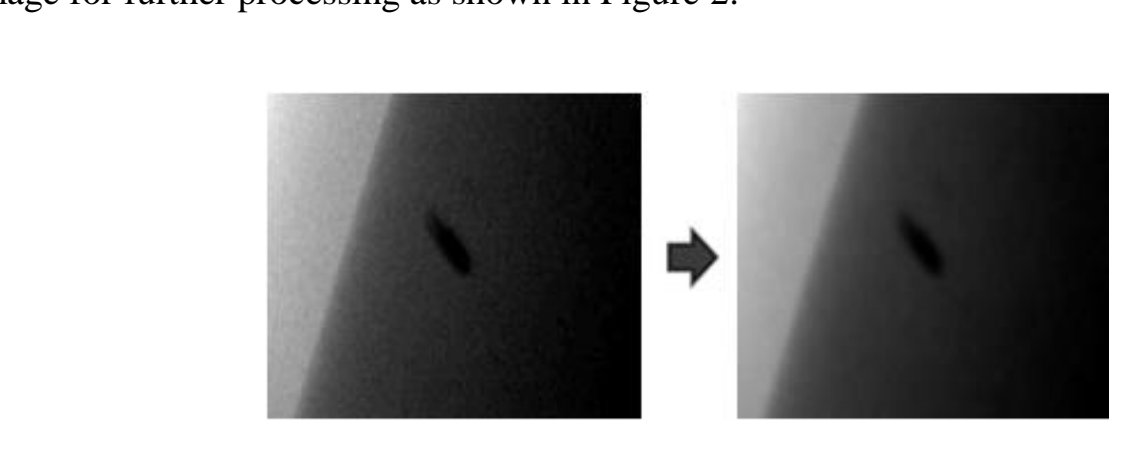

Figure 2. Removing minute noise with mask filtering

\subsection{Extracting Boundary Line with $7 \times 7$ Sobel Mask}

Then the boundary line is extracted by applying with $\mathbf{7} \times \mathbf{7}$ Sobel Mask to the result of 2.2. The vertical and horizontal Sobel mask is as shown in Figure 3. 


\begin{tabular}{|c|c|c|c|c|c|c|}
\hline .1 & -1 & -1 & 0 & 1 & 1 & 1 \\
\hline .1 & -1 & -1 & 0 & 1 & 1 & 1 \\
\hline .1 & -1 & -1 & 0 & 1 & 1 & 1 \\
\hline$\cdot 2$ & -2 & -2 & 0 & -2 & -2 & -2 \\
\hline .1 & -1 & -1 & 0 & 1 & 1 & 1 \\
\hline-1 & -1 & -1 & 0 & 1 & 1 & 1 \\
\hline .1 & -1 & -1 & 0 & 1 & 1 & 1 \\
\hline
\end{tabular}

(a) Vertical Mask



(b) Horizontal Mask

Figure 3. 7x7 Sobel mask

Then the effect of this masking is as shown in Figure 4.
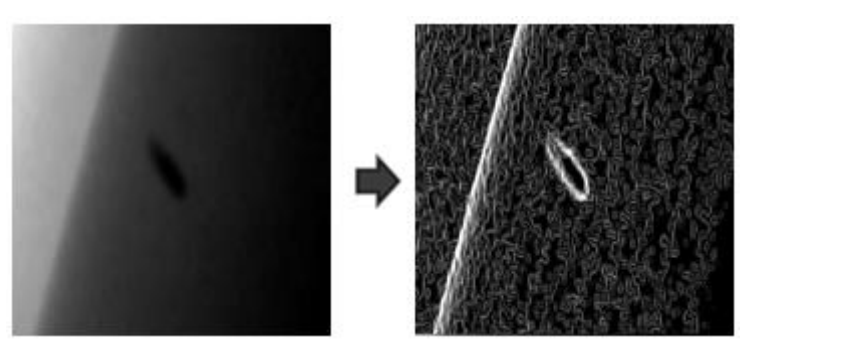

Figure 4. Boundary line after Sobel masking

\subsection{Area Segmentation by Slope Analysis of Boundaries}

Since NDT image has chucial brightness contrast between boundary lines obtained by processes explained above, we compute tbe slope with thresholding binarization and segment areas with that slope as shown in Figure 5.

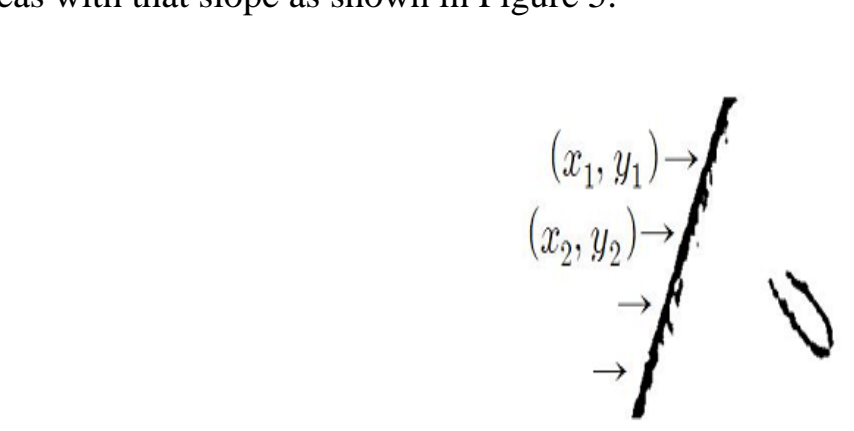

Figure 5. Compute slope from binarized image

In this part, we use different method from previous study [8] in that previous method used the upperleft, lowerleft, upperright, lowerright points in computing the slope but it often recognizes other boundaries or noises as the target boundary line if there does not exist the boundary of interest so that the slope can be miscalculated.

Thus, in this paper, when searching for the boundary area, from $\left(x_{1}, u_{1}\right)$ of the upper part, we try to find $\left(x_{2}, u_{2}\right)$ within an arbitrary range. If that search fails, while the location of $\left(x_{1}, u_{1}\right)$ is fixed, we change the searching range to find $\left(x_{2}, u_{2}\right)$ in computing the slope. Figure 6 compares the effect of this procedure with previous study [8]. 

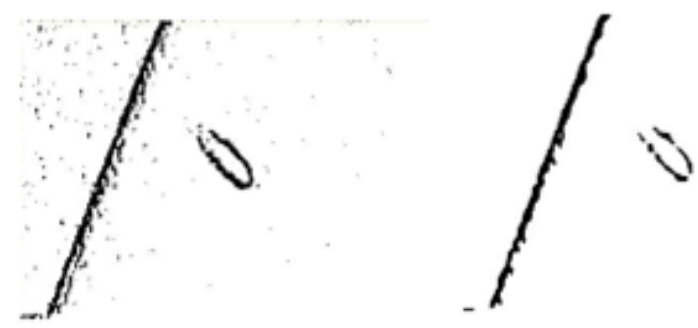

1) Original image
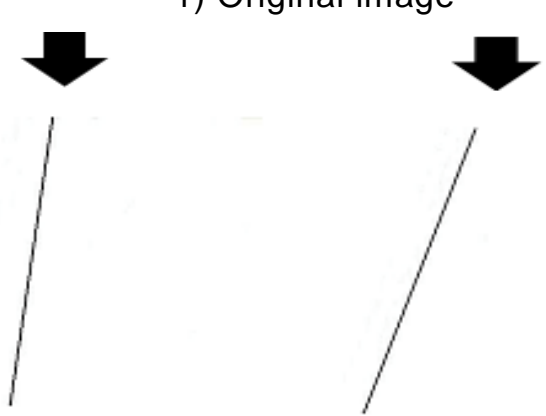

2) Searching the slope

(a) Slope in previous sfudy [8] (b) Slope by proposed method

\section{Figure 6. Area Segmentation}

As one can find in Figure 6) the proposed method is clearly more accurate than previous method. Using that slope, areas are segmented as shown in Figure 7.

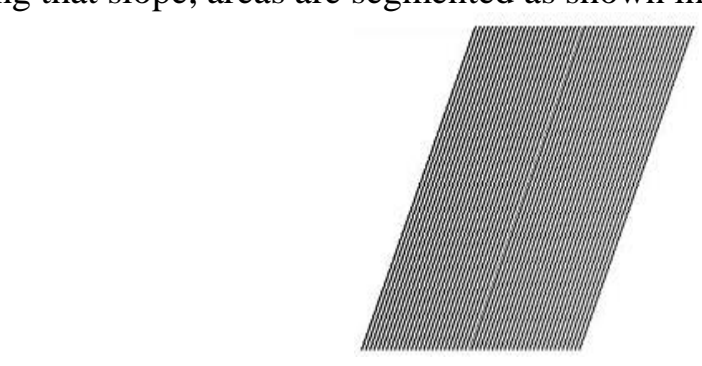

\section{Figure 7. Area segmentation by slope analysis}

\section{Extracting the Defect}

In this part, we discuss how to extract the real defect part from the image segmented by the slope cut explained in Section 2.

\subsection{Area Segmentation by Grassfire labeling}

In order to extract defect area, we would like to apply sigma fuzzy binarization. Then, we need to find a way to compute membership degree of each segmented area for fuzzy membership function and the Grassfire Labeling method [9] is applied for it.

Grassfire labeling searches for pixels that consist of one object with $3 \times 3$ mask and 8directional contour search. First, it searches for a pixel whose brightness is 0 among its 8directions from the pixel whose brightness is equal to the initial mask center. If the search 
succeeds, the center of the mask moves to that pixel and label it, and then take the 8directional search. If the search fails, the center is moved to the previously labeled one. If the initial pixel fails to find pixels to form an object, the next pixel becomes the center.

With that procedure, we label each pixel such that the same object has same valued pixels.

\begin{tabular}{|l|l|l|}
\hline 0 & 0 & 0 \\
\hline 0 & 0 & 0 \\
\hline 0 & 0 & 0 \\
\hline
\end{tabular}

Figure 8. $3 \times 3$ vertical mask for grassfire labeling

The effect of Grassfire labeling is as shown in Figure 9.

\subsection{Extract the Defect by Sigma Fuzzy Binarization}

\section{Figure 9. After)applying grassfire labeling}



In the NDT image, we can find some characteristics that the defect area has different brightness distribution - either brighter or darker - and the size of the defect is relatively small. Thus, the popular binarization methods that use all pixel information of the image is inappropriate to extract defect area.

Therefore, we apply sigma fuzzy binarization method [10] based on the membership degree for segmented areas obtained by previous Section 3.1.

Let Max Min be the highest and lowest brightness value of the pixel from the image obtained by Ends-in Search Stretching and Mid be the average of Max and Min and let abe the quarter of the sum of Max and Min. Then the membership function is defined as the interval $[$ Low,$H i g h]$ and the membership function is defined as follows.

Step 1.

IF Mid $>128$ Then $\alpha=255-$ Mid

ELSE $\alpha=$ Mid

Step 2

Low $=$ Mid $-\alpha$

High $=$ Max

The membership degree of a segmented area computed by sigma fuzzy binarization is as following within interval [Low, High] . 


$$
\begin{aligned}
& I F(\text { Low }>\text { Img AND MId }=0) \text { Then } u(\operatorname{Img})=0 \\
& I F(\text { Low } \leq \operatorname{Img} \leq \text { MId }) \text { Then } u(I m g)=\frac{I m g-\text { Low }}{\text { Mid }- \text { Low }} \\
& I F(\text { Mid }<\text { Img }) \text { Then } u(I m g)=1
\end{aligned}
$$

The membership degree $u(\operatorname{Img})$ is applied to the $\alpha-\operatorname{cut}$ ( set to 0.55 in this paper) for binarization such that the pixel value would be set to 255 if $u(\operatorname{Img}) \geq 0.55$ and 0 otherwise. This binarization process is applied to the image obtained from Ends-in search Stretching . Figure 10 shows the membership function of sigma type.

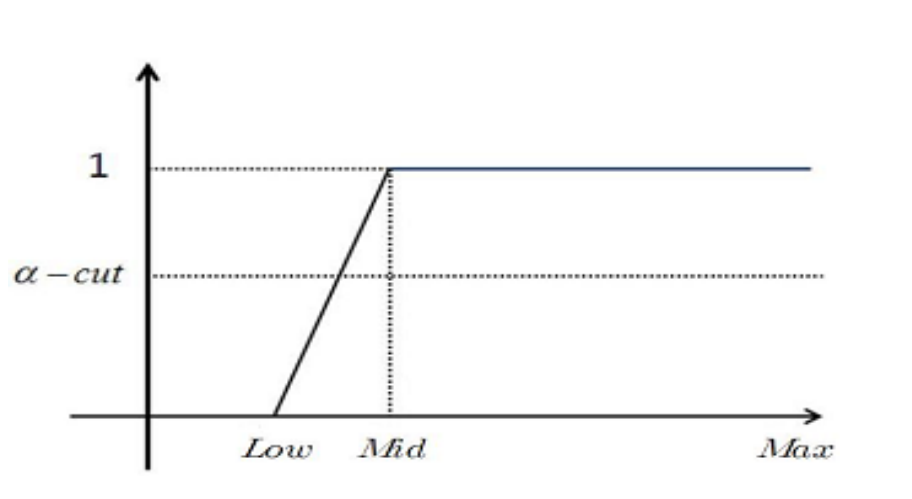

Figure 10. Membership function for sigma fuzzy binarization

Figure 11 shows the result of applying diagonal sigma fuzzy binarization.

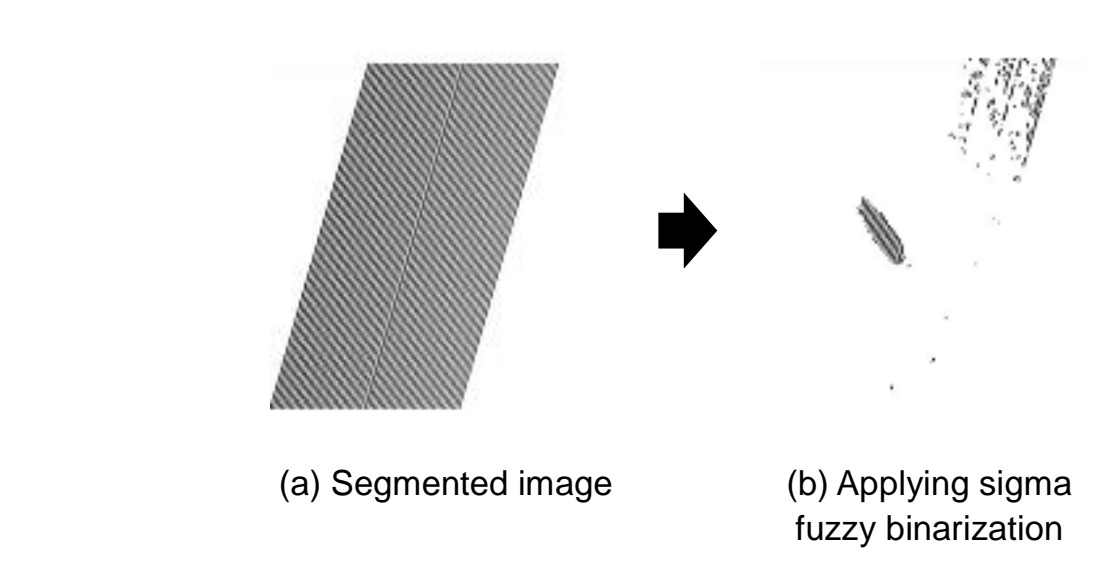

Figure 11. Effect of diagonal sigma fuzzy binarization

\subsection{Removing Noise with Morphological Information}

Still we need to remove minute noises by $3 \times 3$ mask. For each pixel, investigating 8 directions, if pixels with value 255 are more than those of value 0 , the brightness of center pixel is changed to 255 shown as Figure 12. 


\begin{tabular}{|c|c|c|}
\hline 0 & 0 & 255 \\
\hline 0 & 0 & 255 \\
\hline 255 & 255 & 255 \\
\hline
\end{tabular}$\Leftrightarrow$\begin{tabular}{c|c|c|c|}
\hline 0 & 0 & 255 \\
\hline 0 & 255 & 255 \\
\hline 255 & 255 & 255 \\
\hline
\end{tabular}

\section{Figure 12. $3 \times 3$ mask for noise removal}

Figure 13 illustrates the defect extraction process explained in this section as summary.



(a) Grassfire labeling

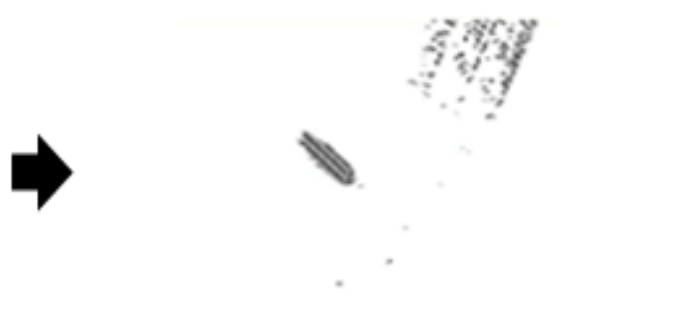

(b) Diagohal sigma fuzzy binarization

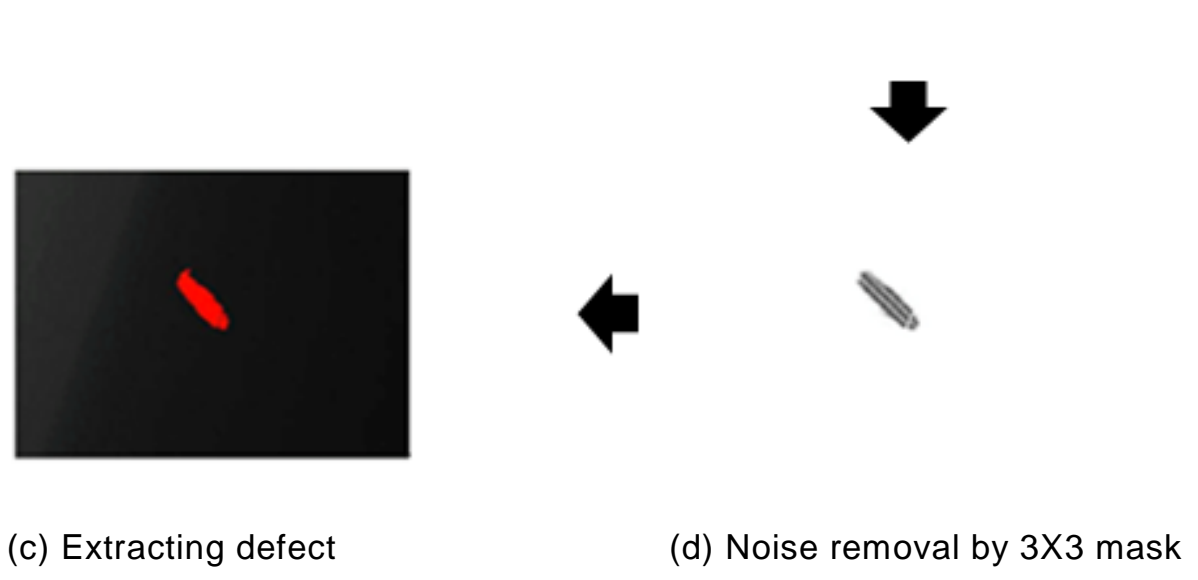

Figure 13. Summary of defect extraction

\section{Experiment and Analysis}

The proposed method is implemented with Microsoft VC++ 6.0 on the IBM-compatible PC with Inel Xeon(TM) CPU 3.20GHz, 2.80GHz and 2GB RAM. The experiment uses ten $640 \times 480$ size NDT images and the results of this automatic defect extraction are shown as Figure 14 and Figure 15.

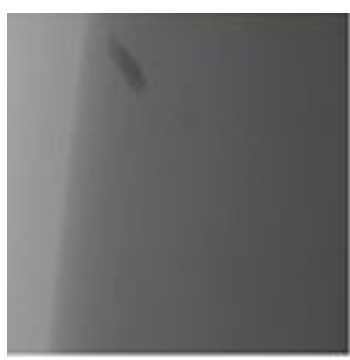

(a) Original Image

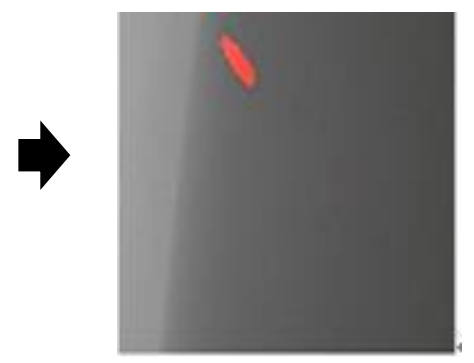

(b) Defect extraction

Figure 14. Example 1 


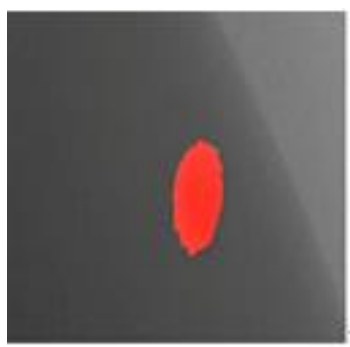

(a) Original image

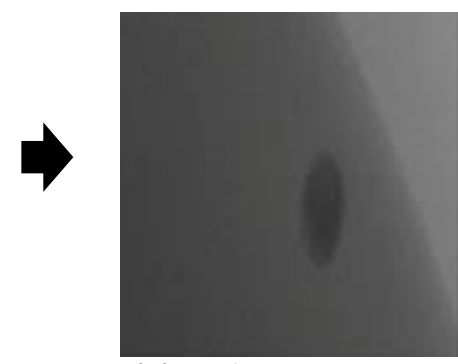

(b) Defect extraction

Figure 15. Example 2

There is another way of verifying the effect of the proposed method. Figure 16-ompares the result of previous method [8] and the proposed method for the same image.



As one an see from Figure 16, the previous method is vulnerable for the input whose brightness distribution is skewed to lower part so the image is darker but the proposed method successfully overcomes the problem. Furthermore, the proposed method is successful to obtain defect area for all six examples as oppose to the incomplete performance of previous method as shown in Table 1.

Table 1. The success fate of defect extraction

\begin{tabular}{|c|c|c|}
\hline & \# of extraction & Success rate \\
\hline Previous[8] & $8 / 10$ & $80 \%$ \\
\hline Proposed & $10 / 10$ & $100 \%$ \\
\hline
\end{tabular}




\section{Conclusion}

In this paper, we propose a method to extract defects on the surface of ceramics from penetrant test(PT) images by computer. This technique can be widely applied to other nondestructive testing methods that require visual inspection for the decision making such that the accuracy of inspection can be more reliable with this type of AI techniques involved.

Many image processing techniques and fuzzy theory are applied to achieve our goal to extract the defect from the PT image. Especially in this paper, we focus on how to handle images that are relatively darker or having uneven brightness distribution. Our newly involved technique - sigma fuzzy binarization and Gaussian Filtering - enable to extract the defect that was failed from our previous study [8].

Regretfully, we did not have sufficient NDT images for this study thus this suecess in experiment might not viewed as a general method to handle PT type NDT images but we would like to assert that this method shows sufficient feasibility for jt. We would like to extend our efforts to handle more various types of defect extraction from/NDT images in general in the future.

\section{References}

[1] http://www.kandt.or.kr

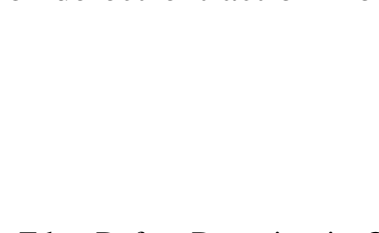

[2] Mansoory, M., Tajik, H., Mohamadi, G., \& Ráshna, M. Edge Defect Detection in Ceramic Tile Based on Boundary Analysis Using Fuzzy Thresholding and Radon Pransform, IEEE International Symposium on Signal Processing and Information Technology, (2008), pp 58-62.

[3] Sarin, C.R., Karthik , M., Anilesh, M Subramaniam, P, "Advanced Image Enhancement of Ultrasonic Scan Images For Intelligent Quality Inspection of Adhesively Bonded Joints in Ceramics", International Journal of Advanced Research in Computer Science and Software Engineering, ( 2012), pp 302-306.

[4] Yong Yang, 'Fuzzy Expectation Maximum Algoithm for Magnetic Resonance Image Segmentation', Journal of Engineering Materials (W. 439 - 440) ( 2010),pp1618-1623.

[5] K. B. Kim, Y. W. Woo "Deteetion of Flaws in Ceramic Materials Using Non-Destructive Testing," Journal of The Korea Institute of Electronic Conmunication Sciences, (2010),Vol.5, No.3, pp.321-326.

[6] J. K. Udupa, G. T Ferman, 3D Imaging in Medicine, CRC Press LLC, (2000).

[7] R.C. Gonzlez, R.E. Woods, Digital Image Processing, Third Edition, Pearson Education, Inc. ( 2008)

[8] K. B. Kim, J. H. Cho, "Detection of Flaws in Air Deck using Non-Destructive Testing," Journal of The Korea Institute of Maritime Infornation \& Communication Sciences ( 2011), Vol.15, No.9, pp.1865-1870.

[9] K. B. Kim, Y. S. Lee, D.F. Yu, A. S. Oh, "Calling Card Recognition using Enhanced RBF Networks, Vol.16, No.2(B), ” INFORMATION-An International Interdisciplinary Journal ( 2013), pp.1517-1522.

[10] K. B. Kim, D. H. Yu, Y.S. Hong, " Extraction of Muscle from Ultrasound Images of Cervical Regions," INFORMATION-An International Interdisciplinary Journal (2013 ), Vol.16, No.4, pp.2669-2678.

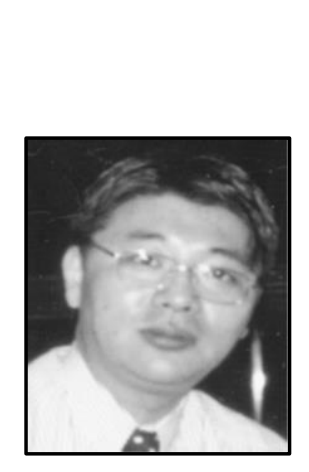

\section{Authors}

Kwang Baek Kim received his M.S. and the Ph.D. degrees in Department of Computer Science from Pusan National University, Busan, Korea, in 1993 and 1999, respectively. From 1997 to present, he is a professor, Department of Computer Engineering, and Silla University in Korea. He is currently an associate editor for Journal of The Korea Society of Computer and Information, and The Open Artificial Intelligence Journal (USA). His research interests include fuzzy neural network and application, bioinformatics and image processing. 


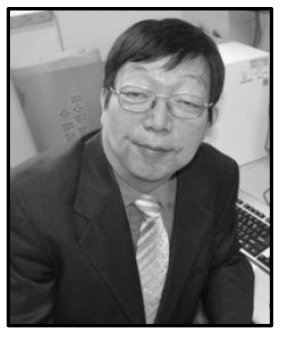

Doo Heon Song received a BS degree in Statistics \& Computer Science from Seoul National University and MS degree Computer Science from the Korea Advanced Institute of Science and Technology in 1983. He received his $\mathrm{PhD}$ Certificate in Computer Science from the University of California in 1994. Form 1983-1986, he was a researcher at the Korea Institute of Science and Technology. He has been a professor at the Department of Computer Games, Songdam College, Korea, since 1997. His research interests include ITS, machine learning, artificial intelligence, medical image processing, cognitive, and game intelligence.

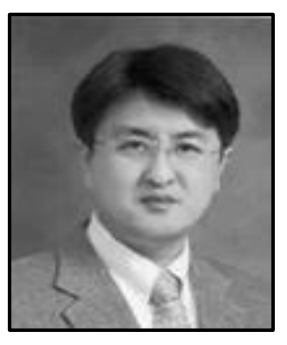

Won Joo Lee received the B.S., M.S. and Ph.D. degrees ip Computer Science and Engineering from Hanyang University(ERICA Campus), Ansan, Korea, in 1989, 1991 and 2004 respectively Dr. Lee joined the faculty of the Department of Computer Science at Inha Technical College, Incheon, Korea, in 2008, where he has served as the Director of the Department of Computer Science. He is currently a Professor in the Department of Computer Science, Inha Technical College. He has also served as the Vice-president of The Korean Society of Computer Information and the Editor-in-Chief for the Journal of The Korean Society of Computer Information. He is interested in parallel computing, internet and mobile computing, and cloud computing.

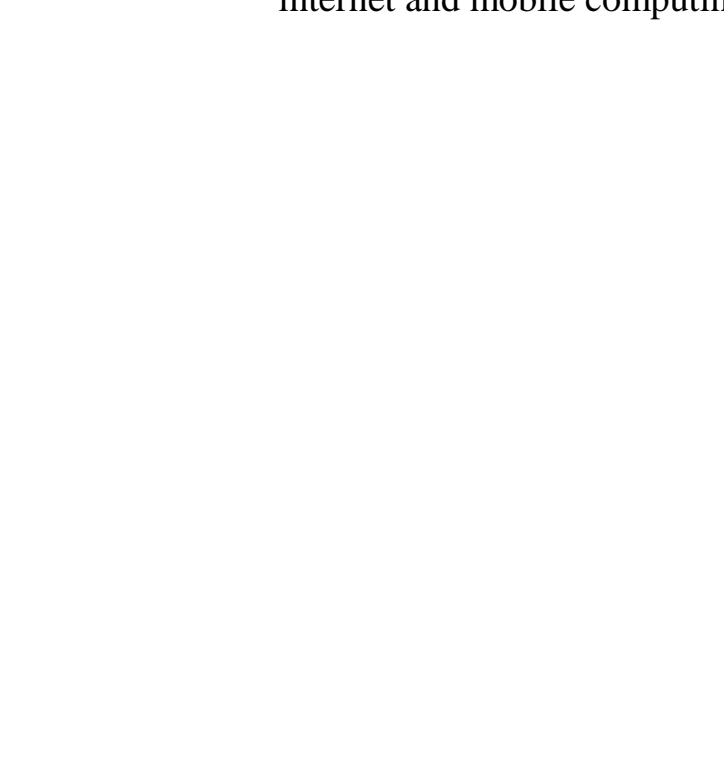


International Journal of Multimedia and Ubiquitous Engineering Vol.9, No.1 (2014)



